\title{
Italian Language
}

National Cancer Institute

\section{Source}

National Cancer Institute. Italian Language. NCI Thesaurus. Code C123788.

A romance language spoken as the official language of Italy and San Marino. 